\title{
Segregación Sectorial por Género: Una Comparación Brasil-México*
}

\author{
Carlos Salas y Marcia Leite**
}

Resumen: El texto examina los cambios en la segregación sectorial por sexo en Brasil y México, entre los años 1995 y 2004. Al tratarse de dos países con un nivel de desarrollo económico semejante, es posible identificar procesos comunes, así como diferencias, lo que nos ayuda a precisar el peso de otros elementos en el nivel de segregación. Para este fin se utiliza un índice, llamado de Karmel-MacLachlan, que permite estudiar la manera en que evoluciona la segregación laboral, a lo largo de un cierto período, por medio de una descomposición de las diferencias entre los valores del índice en dos momentos del tiempo. El índice usa cifras de la estructura de puestos de trabajo según sexo, mismas que provienen de la Pesquisa Nacional Por Amostra Domiciliar (PNAD) de Brasil, y la Encuesta Nacional de Empleo (ENE) de México. Ambas fuentes son encuestas a domicilio con un esquema de muestreo que garantiza su representatividad a escala nacional, y cuyos cuestionarios captan todo tipo de trabajos, así sean marginales.

Palabras-clave: Segregación Laboral, Mercado de Trabajo Mexico, Mercado de Trabajo Brasil.

Abstract: This text analyzes changes in gender segregation by economic sector in Brazil and Mexico, between 1995 and 2004. Since both counties have a similar development level, it is possible to identify common processes, as well as differences, which help to pinpoint the weight other elements have on the level of segregation. To achieve this goal, the paper uses the Karmel-MacLachlan index, which allows for a decomposition of the changes in segregation index levels in two poitns of time. We use survey data from Brazil's Pesquisa Nacional Por Amostra Domiciliar (PNAD) de Brazil, and Mexico's Encuesta Nacional de Empleo (ENE). Both are representative at national level and have information on all kind of jobs, including marginal.

Keywords: Segregation Labor, Labor Market Mexico, Labor Market Brazil.

\footnotetext{
* Una versión más breve será publicada en el libro Marché du travail et genre. Comparaisons internationales France-Brésil, Editions La Decouverte

** Carlos Salas, Colegio de Tlaxcala, México. E-mail: csalasp51@gmail.com. Márcia Leite, UNICAMP, Brasil. E-mail: mpleite@uol.com.br E-mail: Recebido em 26/04/07 e aceito em 10/12/07.
} 


\section{Introducción}

A pesar de una mayor participación en el trabajo extradoméstico remunerado, las condiciones y niveles de pago promedio de las mujeres exhiben una persistente diferencia respecto a las de los hombres. Y, aunque la brec, ha viene disminuyendo en los últimos años, esta persiste. Tal fenómeno no es exclusivo de un país en particular, aunque la magnitud de la brecha varía mucho entre países. En la teoría social se ha propuesto la idea de que la diferencia de ingresos es el resultado de la acción de, al menos dos factores: la discriminación abierta, y la forma en que hombres y mujeres se distribuyen entre las diversas ocupaciones o sectores económicos de actividad. Es un hecho fácilmente verificable que existen actividades donde se concentran, de manera preferente, los trabajadores de uno y otro sexo. A este fenómeno se le conoce con el nombre de segregación ocupacional, o segregación sectorial, por sexo, según se trate de la manera en que hombres y mujeres se distribuyen entre diversas ocupaciones específicas o entre diversos sectores de actividad.

En este texto vamos a concentrarnos en examinar los cambios en la segregación sectorial por sexo en Brasil y México, entre los años 1995 y 2004. Al tratarse de dos países con un nivel de desarrollo económico semejante, es posible identificar procesos comunes, así como diferencias, lo que nos ayuda a precisar el peso de otros elementos en el nivel de segregación.

Para este fin se utiliza un índice, llamado de Karmel-MacLachlan, que permite estudiar la manera en que evoluciona la segregación laboral, a lo largo de un cierto período, por medio de una descomposición de las diferencias entre los valores del índice en dos momentos del tiempo. El índice usa cifras de la estructura de puestos de trabajo según sexo, mismas que provienen de la Pesquisa Nacional Por Amostra Domiciliar (PNAD) de Brasil, y la Encuesta Nacional de Empleo (ENE) de México. Ambas fuentes son encuestas a domicilio con un esquema de muestreo que garantiza su representatividad a escala nacional, y cuyos cuestionarios captan todo tipo de trabajos, así sean marginales.

El texto está dividido en tres apartados. En el primero se define el concepto de segregación ocupacional y se hace una breve discusión sobre las causas y consecuencias de la misma, así como sobre su medición.

El segundo inicia con una rápida descripción de la división del trabajo 
por sexo para Brasil y México. Posteriormente se presentan y discuten los resultados de aplicar la técnica referida a la distribución de los trabajadores - hombres y mujeres- entre sectores de la actividad económica, para cada país, en el período 1995-2004.

El tercer apartado se dedica a llevar a cabo algunas comparaciones entre los dos países.

\section{Segregación ocupacional por sexo: orígenes y consecuencias}

Después de un proceso sostenido de aumento en la tasa de actividad de las mujeres entre 1970 y 1990, a escala mundial, esta tasa ha tendido a incrementarse solo de manera marginal entre 1996 y 2006. Pero en América Latina y el Caribe, el incremento sigue siendo importante, aún entre 1996 y 2006 (OIT, 2007), aunque esta mayor participación laboral no se ha traducido en una mejoría sustantiva de las condiciones laborales de la mujer. El informe de la OIT referido señala que la segregación laboral por sexo ha disminuido muy poco durante el período de análisis.

Estos hechos conducen a una serie de cuestiones, las cuales se pueden englobar en tres grandes grupos:

1. Causas de la segregación y las diferencias salariales

2. Explicaciones relativas a la persistencia de ambos fenómenos

3. Efectos posibles de la interacción entre ellos.

En la literatura de ciencias sociales hay diversas interpretaciones tanto del origen de la segregación como de las diferencias salariales entre hombres y mujeres. Las discrepancias surgen por la existencia de dos perspectivas analíticas opuestas. Una de ellas, el llamado individualismo metodológico, concibe a todos los procesos sociales como explicables sólo a partir de decisiones individuales, mientras que la otra, llamada genéricamente holismo metodológico, explica esos mismos procesos sociales en términos de estructuras sociales, de instituciones sociales o de la cultura. Para la primera, los precios son el principal mecanismo de interacción social de los individuos, mientras que la segunda privilegia el contexto social donde estas interacciones económicas tienen lugar.

Como es bien sabido, la vertiente neoclásica en la economía (y su 
contraparte en la sociología, la escuela de la elección racional) tiene su fundamento en el individualismo metodológico, mientras que las vertientes heterodoxas parten de considerar la importancia del contexto social como el telón de fondo. Las posturas teóricas basadas en el individualismo metodológico, sostienen que las diferencias de ingreso y la segregación son el resultado de decisiones de los individuos, que se expresan en la forma de diferentes "niveles de inversión en capital humano", según se trate de hombres o mujeres, o en diversas formas de discriminación de los empleadores ${ }^{1}$.

La teoría social apoyada en el individualismo metodológico carece del concepto de género, puesto que éste último está basado en la idea de que los roles masculinos o femeninos son características sociales. Tal ausencia tiene consecuencias inesperadas. Así, al estudiar los ingresos de las mujeres, algunos resultados empíricos obtenidos por autores de inspiración neoclásica, plantean verdaderas paradojas, que serían perfectamente explicables a partir de un análisis de género. El hecho de que la teoría social apoyada en el individualismo metodológico carezca del concepto de género, puesto que éste último está basado en la idea de que los roles masculinos o femeninos son características sociales, tiene consecuencias inesperadas. Así, al estudiar los ingresos de las mujeres, algunos resultados obtenidos por autores de inspiración neoclásica, plantean verdaderas paradojas para esa visión teórica. Por ejemplo, Fogel (2006) encuentra que, en el caso de Brasil, una mayor feminización de las empresas, está asociada a menores niveles de ingreso, tanto de hombres como mujeres ${ }^{2}$. Este resultado es fácilmente explicable usando la noción de ocupaciones tipificadas por sexo, y sin embargo, en las conclusiones del artículo referido, el autor afirma que entre las razones detrás del hecho estudiado se encuentran ¡“características no medidas de las mujeres" (OLIVEIRA, 2001, p. 62), debido a que no existen elementos dentro de su visión teórica que le permitan explicar el que algunas personas elijan ocupaciones tipificadas por sexo. Otro caso aparece en Vella (1993), quien usando una encuesta australiana que capta información sobre historia laboral y actitudes respecto de los roles tradicionales de las mujeres, termina por encontrar la obviedad de que una actitud menos "tradicional" de las

\footnotetext{
${ }^{1}$ Una presentación reciente de este tipo de argumentos se puede encontrar en (FOGEL, 2006)

${ }^{2}$ Este resultado ya había sido señalado, entre otros, por Oliveira (2001).
} 
mujeres respecto de su papel social, está acompañada de un mayor ingreso laboral.

Por otro lado, las explicaciones de corte holístico, en especial las originadas en el feminismo, plantean que la segregación está sustentada en la construcción social denominada género, que hace que ciertas actividades sean consideradas como tipificadas por sexo (ENGLAND, 1992; JACOBS, 2001). Al ser considerada una ocupación como "femenina" o "masculina”, se obtiene un menor o mayor ingreso por ese tipo de trabajo.

Es posible que en un mismo lugar de trabajo se pague menos a las mujeres que a los hombres cuando desempeñan las mismas tareas, sin embargo, tal práctica es cada vez menos común debido a que la disposición legal que establece el "pago igual a trabajo igual" tiende a generalizarse en el mundo. Esta disposición se encuentra en la Convención Número 100 de la Oficina Internacional del Trabajo, adoptada en 1953, a la que se adhieren los estados miembros de la OIT. En consecuencia, la explicación de la desigualdad salarial entre los sexos debe buscarse en otra parte, a saber en la segregación ocupacional (sectorial) por sexo, misma que definimos en seguida.

Si hombres y mujeres se distribuyeran al azar entre sectores y ocupaciones, entonces la proporción de hombres (mujeres) en cada ocupación (o sector) debería ser igual a la proporción que los hombres (mujeres) representan del personal ocupado total. Cuando esta proporción es distinta, se dice que hay segregación ocupacional (o sectorial) por sexo, Anker (1998) y Watts (1998).

A lo largo del tiempo, el nivel de segregación tiende a reducirse. El cambio suele ser lento, pero en ocasiones, los niveles de segregación pueden alterarse en forma notable. Esto lo muestran algunos estudios referidos principalmente a países industrializados (HAKIM, 1994). Aunque es de observarse que no existen investigaciones semejantes para América Latina, vale destacar algunos trabajos importantes a nivel nacional, como el de Bruschini y Lombardi, (2003) para el caso de Brasil y el Maldonado y Rendón (2005) para el caso de México.

La segregación laboral por sexo, entre ocupaciones específicas o sectores de actividad económica, es un fenómeno que no basta ser estudiado examinando, caso por caso, la manera en que los sexos se distribuyen entre 
cada ocupación o sector de actividad. Para dar cuenta del fenómeno en su totalidad, existe un variado conjunto de índices que representan una medida sumaria de la manera en que hombres y mujeres se distribuyen entre diversas ocupaciones específicas o entre los distintos sectores de actividad ${ }^{3}$. En la literatura especializada puede encontrarse una fuerte discusión sobre las propiedades, utilidad y validez de muchos de ellos, entre estos el índice de disimilitud de Duncan ${ }^{4}$, mismo que ha sido empleado en el Brasil para medir la segregación de ocupaciones por sexo ${ }^{5}$. El índice puede interpretarse como el porcentaje de mujeres (y hombres) que debe cambiar de ocupación para que la distribución de empleo de los dos grupos sea igual.

Sin embargo, debido a las dificultades que surgen para explicar la evolución del índice de disimilitud a lo largo del tiempo se han propuesto otros índices, que buscan resolver algunos de estos problemas (BLACKBURN et al. 1995; CARLSON, 1992; KARMEL y MACLACHLAN, 1988; WATTS 1992 y 1998). Pero como se muestra en Salas (2004), salvo el índice que se propone en el artículo de Karmel y Maclachlan, los demás están llenos de problemas de interpretación y no captan correctamente la distribución desigual de hombres y mujeres entre ocupaciones (o sectores de actividad).

Debido a sus propiedades, en particular, por la posibilidad de descomponer el índice entre dos puntos en el tiempo, aquí vamos a utilizar el índice desarrollado por Karmel y Maclachlan', y que denotaremos por KM.

$\mathrm{La}$ interpretación de $\mathrm{KM}$ es directa, se trata del nivel total del empleo que debe reasignarse (con reemplazo) para conseguir una segregación por sexo igual a cero, pero manteniendo la estructura sectorial y la proporción de mujeres en el empleo total. Obsérvese el contraste de esta interpretación con la correspondiente al índice de Duncan.

En general, el valor de un índice de segregación puede variar a consecuencia

\footnotetext{
${ }^{3}$ Ver en particular Anker (1998) y Salas (2004).

${ }^{4}$ El índice se calcula de la manera siguiente: suponiendo que existen n ocupaciones distintas, sea hi=Hi/H la proporción de hombres que tienen la ocupación i, y de manera análoga, $m_{i}=M_{i} / M$ la proporción de mujeres en la ocupación i. Entonces $D=\frac{1}{2} \sum_{i=1}^{n}\left|h_{i}-m_{i}\right| * 100$. Cuando ID $=0$ no hay segregación, y si ID $=100$ entonces hay una separación absoluta entre las ocupaciones que realizan los hombres y las mujeres (Duncan y Duncan, 1955).

${ }^{5}$ Ver, por ejemplo, Oliveira (2001).

${ }^{6}$ El índice KM es 2a (1-a)*ID, donde ID es el índice de dismilitud de Duncan y a es la proporción de hombres en el total de la fuerza de trabajo.
} 
de diversos factores. En Karmel y MacLachlan (1988), se demuestra cómo los cambios en el índice KM a través del tiempo, se pueden separar en un efecto composición y en un efecto mixto. El efecto composición mide el cambio en la proporción de sexos en cada sector de actividad, manteniendo el empleo total y los niveles de empleo femenino y masculino constantes. Mientras que el efecto mixto es una medida del cambio en el índice que se puede atribuir al cambio en las proporciones de sexos y proporciones de cada sector en el empleo total, manteniendo constante la composición de sexos de cada sector. A su vez el efecto mixto se puede descomponer en efectos de sector, sexo y de interacción. En este caso, se tiene en primer lugar, el cambio originado en modificaciones en la estructura sectorial, manteniendo constante la composición por sexo de la fuerza de trabajo total (efecto sector); en segundo lugar, el cambio debido a modificaciones en la proporción de hombres y mujeres en la fuerza de trabajo total, manteniendo constante la estructura sectorial (efecto sexo); y en tercer lugar, un residuo que se atribuye a la combinación de los dos anteriores (efecto sector-sexo). Si bien, en este texto, examinamos la segregación laboral por sector, es perfectamente posible hacer el mismo análisis por ocupaciones específicas.

\section{Segregación laboral por sectores en Brasil y México, 1995-2004}

\section{El Caso de Brasil}

Los estudios sobre las relaciones de género en el mercado de trabajo brasileño evidencian una serie de tendencias las cuales, si bien apuntan a mejorías significativas de las condiciones de trabajo, también señalan el mantenimiento de formas de desigualdad que parecen resurgir una y otra vez. Esas tendencias que ya parecían claras en la década de los 90 (BRUSCHINI, LOMBARDI, 2003) se mantienen en los años 2000.

La Tabla 1 exhibe la composición por sexo de los sectores de actividad entre 1995 y 2004 y sirve de base para calcular el índice de segregación. Además de mostrar que hay un aumento en la proporción de mujeres en el total de puestos de trabajo, nos permite explicar, tal como se verá más adelante, los cambios observados en el índice de segregación. 
Tabla 1 - Población ocupada de 10 y más años y proporción de mujeres dentro del empleo total, por sector de actividad y sexo. Brasil: 1995-2004

\begin{tabular}{|c|c|c|c|c|c|c|}
\hline & \multicolumn{3}{|c|}{1995} & \multicolumn{3}{|c|}{2004} \\
\hline & Hombres & Mujeres & $\begin{array}{l}\text { Proporción } \\
\text { mujeres en } \\
\text { el empleo } \\
\text { total }\end{array}$ & Hombres & Mujeres & $\begin{array}{l}\text { Proporción } \\
\text { mujeres en } \\
\text { el empleo } \\
\text { total }\end{array}$ \\
\hline Agrícola & 11907665 & 6246577 & 0.344 & 12062961 & 5670874 & 0.320 \\
\hline $\begin{array}{l}\text { Industria de la } \\
\text { transformación }\end{array}$ & 6212882 & 2335518 & 0.273 & 7370015 & 4353625 & 0.371 \\
\hline $\begin{array}{l}\text { Otras actividades } \\
\text { industriales }\end{array}$ & 717607 & 145192 & 0.168 & 602684 & 76368 & 0.112 \\
\hline Construcción & 4125314 & 103891 & 0.025 & 5220131 & 134244 & 0.025 \\
\hline $\begin{array}{l}\text { Comercio y } \\
\text { reparación }\end{array}$ & 7477139 & 3644429 & 0.328 & 9043910 & 5609318 & 0.383 \\
\hline $\begin{array}{l}\text { Alojamiento y } \\
\text { alimentación }\end{array}$ & 1359904 & 1108042 & 0.449 & 1518415 & 1504644 & 0.498 \\
\hline $\begin{array}{l}\text { Transporte, } \\
\text { almacenaje y } \\
\text { comunicación }\end{array}$ & 2411886 & 245888 & 0.093 & 3428309 & 465940 & 0.120 \\
\hline $\begin{array}{l}\text { Actividades } \\
\text { financieras e } \\
\text { inmobiliarias }\end{array}$ & 1469283 & 971838 & 0.398 & 1162676 & 704306 & 0.377 \\
\hline $\begin{array}{l}\text { Educación, salud y } \\
\text { servicios sociales }\end{array}$ & 1516906 & 4527094 & 0.749 & 1724483 & 5783541 & 0.770 \\
\hline Otros servicios & 1552162 & 2041533 & 0.568 & 3829651 & 3422371 & 0.472 \\
\hline Servicios domésticos & 760080 & 5277895 & 0.874 & 432417 & 6040067 & 0.933 \\
\hline $\begin{array}{l}\text { Administración } \\
\text { pública y organismos } \\
\text { internacionales }\end{array}$ & 2144273 & 1072110 & 0.333 & 2638892 & 1569026 & 0.373 \\
\hline $\begin{array}{l}\text { Otras actividades, } \\
\text { mal definidas o no } \\
\text { declaradas }\end{array}$ & 208208 & 45292 & 0.179 & 207431 & 19995 & 0.088 \\
\hline Total & 41863309 & 27765299 & 0.399 & 49241975 & 35354319 & 0.418 \\
\hline
\end{tabular}

FUENTE: Cálculos propios a partir de los datos individuales de la PNAD

$\mathrm{Al}$ analizar los datos relativos a la participación de las mujeres en la PEA y sus ingresos laborales en el período entre 1995 y 2004, la PNAD indica claramente que la participación de la mujer sigue aumentando, al pasar de una tasa del 48.1 al $52.9 \%$, y que la desigualdad de los ingresos continúa cayendo, ya que la proporción de los ingresos de las mujeres, respecto de los hombres, pasa del 76 al $82 \%$. 
El aumento de la participación femenina ocurre solamente para los grupos de edad entre los veinte y los 59 años $^{7}$. Lo anterior indica un cambio significativo en la conducta de las mujeres, quienes cada vez menos consideran incompatibles sus actividades domésticas con el trabajo profesional. Este fenómeno se puede explicar, parcialmente, como una respuesta de las mujeres a la pérdida de empleo que los hombres sufren en el período considerado, el cual se expresa en menores tasas de actividad para ellos, ya que entre 1995 y 2004 , dichas tasas caen del 75,3 al 73,6\%. Pero, es indudable que otra parte de la explicación reside en un cambio en la conducta de las mujeres encaminada ahora hacia la independencia y autonomía que se expresa en otros datos, como son su mayor nivel de escolaridad cuando comparado al de los hombres, así como el crecimiento de las familias monoparentales que tienen las mujeres como jefas.

Respecto de la remuneración al trabajo femenino, hay que señalar que, al tomar el período 1995-2004 en su conjunto, se pierde un proceso importante, el cual hemos analizado en otro artículo (POCHMAN, SALAS, LEITE, 2007), que se refiere al reciente quiebre de la tendencia hacia la baja en los niveles de ingreso, tanto de hombres como de mujeres a partir de 1999.

Varios estudios ya han señalado que el mayor ingreso laboral de los hombres, respecto de las mujeres, contrasta con los mayores niveles de escolaridad relativa de las mujeres (entre otros ver Cacciamali e Hirata, 2005). Este hecho remite a la discusión relativa a la segregación de las mujeres en actividades donde el trabajo es menos valorizado (como es el caso de las actividades del sector terciario), en cuanto los hombres representan la mayoría de los trabajadores del sector secundario. Asimismo, las mujeres se concentran en actividades que remiten al imaginario social de que el trabajo de cuidar constituye una actividad eminentemente femenina (profesoras, enfermeras) ${ }^{8}$.

La segregación ocupacional remite no sólo a políticas empresariales de contratación, sino también a la búsqueda que las mujeres realizan preferiblemente en actividades y a carreras identificadas con tal imaginario, lo

\footnotetext{
${ }^{7}$ Según apunta el estudio de Bruschini y Lombardi (2003) también es significativa para las mujeres con hijos, inclusive para aquellas que tienen hijos de menos de dos años.

${ }^{8}$ Para una visión de cómo un mismo estereotipo de género aparece en diversos páses, ver Adler (1993). El texto incluye capítulos sobre Brasil y México.
} 
cual promovería la tipificación por sexo de las ocupaciones, y la consecuente formación de guetos profesionales masculinos y femeninos (BRUSCHINI, LOMBARDI, 2003). Dicha tipificación recorre, de punta a punta, el mercado de trabajo. Envuelve todo tipo de actividades, desde las más simples, que demandan menor grado de calificación y formación, hasta las relacionadas con la enseñanza universitaria, donde encontramos una mayor concentración de hombres en las áreas de la ingeniería y la tecnología, mientras que las mujeres están concentradas en las ciencias humanas, biológicas y de la salud (BRUSCHINI, LOMBARDI, 2003).

En (POCHMAN, SALAS, LEITE, 2007) ya habíamos encontrado que entre 2001 y 2004, la segregación por sectores según sexo -medida con el índice de Karmel-Maclachlan- había disminuido. Ahora examinamos con más detalle la segregación laboral por sectores, entre 1995 y 2004. Para este fin calculamos el índice KM para medir la segregación laboral por sectores de actividad. Después, usando la técnica comentada anteriormente, se desagrega el cambio total del índice en varias componentes. En primer lugar, un efecto composición y en segundo un efecto mixto. A su vez, el efecto mixto se desagrega en un efecto sector, un efecto sexo y un efecto combinado sectorsexo. Los resultados del ejercicio aparecen en la Tabla 2.

Tabla 2 - Indice de Karmel y MacLachlan (KM) de segregación de sexos por sectores de actividad, 1995 y 2004 y descomposición del cambio en el índice Brasil
A) KM 1995
16.430
B) KM 2004
15.640
C) Cambio total del índice (A-B)
$-0.793$
Descomposición del cambio: $\mathrm{C}=\mathrm{D}+\mathrm{E}$
D) Efecto composición
8.851
E) Efecto mixto
$-9.644$
Sector
0.377
Sexo
$-9.490$
Sector-Sexo

FUENTE: Cálculos propios a partir de los resultados de la PNAD de 1995 y 2004.

Como muestra la Tabla 2, la segregación disminuye en 0.793. La caída en la segregación se debe, sobre todo al efecto mixto, esto es, a las modificaciones de la estructura sectorial que fueron acompañadas de una mayor participación de las mujeres en las actividades económicas. 
A partir de las cifras que aparecen en la Tabla 1, se comprueba que los sectores que mas contribuyeron (positiva o negativamente) al cambio en el total de mujeres, fueron el Agrícola, donde se observó una baja en la participación de las mujeres; la Manufactura, sector en el cual la participación femenina se incrementó, y el sector Comercio y Reparación, donde la proporción de fuerza de trabajo femenina tuvo también un aumento significativo.

\section{El caso de México}

Pasamos ahora a examinar el caso de México. Hay una vasta literatura que muestra una creciente absorción de fuerza de trabajo femenina,. Sin embargo, ésta es todavía muy reducida: Más del 60\% de las mujeres no realizan actividades económicas fuera de su hogar, además de que las mujeres trabajan, en promedio, menos horas que los hombres (MALDONADO, RENDÓN, 2005). Como veremos enseguida, persiste la segregación laboral por sexo, si bien ésta tiende a decrecer de manera lenta.

La Tabla 3 nos muestra la estructura por sexos del empleo por sector de actividad económica y la proporción de mujeres en cada sector. Los resultados indican un lento, pero constante, aumento de la participación femenina en el empleo, que se concentra en las actividades de comercio y servicios. Sin embargo destaca el hecho de que la participación de las mujeres en las actividades de manufactura tiene un importante aumento, ya que su número casi se duplica en diez años. En contraste, el sector de actividades financieras, sector ya masculinizado, tiene un crecimiento significativo de la participación masculina en este período. 
Tabla 3 Población ocupada de 12 y más años y proporción de mujeres dentro del empleo total, por sector de actividad y sexo. México: 1995-2004

\begin{tabular}{|c|c|c|c|c|c|c|}
\hline & 1995 & & & 2004 & & \\
\hline & Hombres & Mujeres & $\begin{array}{l}\text { Proporción } \\
\text { mujeres en } \\
\text { el empleo } \\
\text { total }\end{array}$ & Hombres & Mujeres & $\begin{array}{l}\text { Proporción } \\
\text { mujeres en } \\
\text { el empleo } \\
\text { total }\end{array}$ \\
\hline Agropecuarias & $6,770,994$ & $1,102,763$ & 0.140 & $5,862,642$ & 858,031 & 0.128 \\
\hline Minas y canteras & 101,105 & 17,850 & 0.150 & 92,069 & 2,462 & 0.026 \\
\hline $\begin{array}{l}\text { Extracción y } \\
\text { Refinación de } \\
\text { Petróleo }\end{array}$ & 103,945 & 18,355 & 0.150 & 118,960 & 32,166 & 0.213 \\
\hline $\begin{array}{l}\text { Industria de } \\
\text { Transformación }\end{array}$ & $3,466,202$ & $1,504,109$ & 0.303 & $4,478,176$ & $2,698,857$ & 0.376 \\
\hline Electricidad & 67,411 & 12,061 & 0.152 & 199,315 & 40,046 & 0.167 \\
\hline Construcción & $1,722,927$ & 49,686 & 0.028 & $2,618,089$ & 86,493 & 0.032 \\
\hline Comercio & $3,115,535$ & $2,989,400$ & 0.490 & $4,059,062$ & $3,938,510$ & 0.492 \\
\hline $\begin{array}{l}\text { Hoteles, } \\
\text { Restaurantes y } \\
\text { Similares }\end{array}$ & 709,181 & 790,460 & 0.527 & $1,005,564$ & $1,308,628$ & 0.565 \\
\hline $\begin{array}{l}\text { Transportes y } \\
\text { Servicios Conexos }\end{array}$ & $1,237,516$ & 84,732 & 0.064 & $1,594,893$ & 99,449 & 0.059 \\
\hline Comunicaciones & 84,598 & 39,318 & 0.317 & 117,976 & 66,721 & 0.361 \\
\hline $\begin{array}{l}\text { Alquiler de } \\
\text { Inmuebles y } \\
\text { Servicios Financieros } \\
\text { y Profesionales }\end{array}$ & 692,751 & 405,604 & 0.369 & $1,172,646$ & 630,557 & 0.350 \\
\hline Otros Servicios & $3,217,230$ & $3,138,826$ & 0.494 & $4,251,455$ & $4,335,004$ & 0.505 \\
\hline $\begin{array}{l}\text { Administración } \\
\text { Pública y Defensa }\end{array}$ & 895,461 & 377,196 & 0.296 & $1,207,784$ & 601,631 & 0.333 \\
\hline No especificado & 131,472 & 19,477 & 0.129 & 127,652 & 33,883 & 0.210 \\
\hline TOTAL & $22,316,328$ & $10,549,837$ & 0.321 & $26,906,283$ & $14,732,438$ & 0.354 \\
\hline
\end{tabular}

FUENTE: INEGI, Encuesta Nacional de Empleo y cálculos propios

Las tasas de actividad entre 1995 y 2004 , para las mujeres fueron de 34,5 y $35,2 \%$, respectivamente, mientras que en el caso de los hombres, las cifras correspondientes fueron de 78,2 y 74,6\%. Un análisis más detallado muestra, en primer lugar, que la caída en la participación de los hombres, en especial entre los menores de 40 años, fue acentuada por las bajas en el nivel 
de actividad entre los 12 y los 24 años. Este fenómeno es la combinación de mayores niveles de participación escolar y una salida de la fuerza de trabajo debido a las mayores dificultades para encontrar empleo. En contraste, las tasas de actividad de las mujeres se vieron incrementadas, sobre todo en los grupos de edad entre los 25 y 60 ańos. El grupo de edad entre los 12 y los 24 redujo su nivel de participación laboral, en parte por un involucramiento mayor con las actividades escolares

Ahora examinamos como evolucionó el pago al trabajo en el período 1995-2004. Hay que recordar que la crisis de 1995, representó una fuerte pérdida del poder adquisitivo de los ingresos por trabajo, de manera que la comparación parte de niveles de ingreso real muy reducidos. La proporción de ingresos por hora de las mujeres respecto de los hombres y mujeres pasó de .99 a 1, entre los años 1995 y 2004.

Sin embargo, el ingreso promedio mensual de hombres y mujeres sigue siendo muy desigual, debido a que las mujeres trabajan menos horas que los hombres: Así, en 2004, el ingreso promedio mensual de las mujeres representaba apenas el 83\% del ingreso de los hombres. Además, en aquellas actividades típicamente masculinas, como es el caso de la industria extractiva, las mujeres tienden a ocupar puestos administrativos, lo que incrementa su nivel de ingreso, en comparación con los hombres, quienes se ubican en todo tipo de ocupaciones. De manera que estos resultados deben ser relativizados.

Pasamos a presentar los resultados de la estimación del grado de segregación sectorial por sexo, para 1995 y 2004, así como de la descomposición de los cambios ocurridos, mediante las técnicas usadas en el caso de Brasil. 
Tabla 4 - Indice de Karmel y MacLachlan (KM) de segregación de sexos por sectores de actividad, 1995 y 2004 y descomposición del cambio en el índice. México
A) KM 1995
15.155
B) KM 2004
14.681
C) Cambio total del índice (A-B)
$-0.474$
Descomposición del cambio: $\mathrm{C}=\mathrm{D}+\mathrm{E}$
D) Efecto composición
E) Efecto mixto
0.017

\begin{tabular}{|l|l|}
\hline Sector & -0.421 \\
\hline Sexo & -5.198 \\
\hline Sector-Sexo & 5.636 \\
\hline
\end{tabular}

FUENTE: Cálculos propios a partir de los resultados de la ENE del segundo trimestre de 1995 y 2004.

La caída en el nivel de segregación por sector, fue muy reducida, debido al efecto combinado de dos procesos. En primer lugar, la participación de la mujer en las actividades económicas aumentó sobre todo en las actividades de servicios, donde la presencia femenina ya era importante. Por tanto, este factor contribuyó a dejar la segregación casi constante. Pero el aumento considerable de la participación femenina en las actividades de la manufactura, incidió en la baja en el nivel de segregación.

\section{Brasil y México en comparación}

Brasil es el mayor país de América Latina. Las cifras que siguen, referidas a 2004 muestran algunos rasgos significativos. La población estimada era de 183.9 millones de personas. Mientras que el PIB fue de 6637.6 millones de dólares, y el ingreso per cápita fue de 3320 dólares.

Entre 1990 y 2005, el ingreso per cápita creció a una tasa media anual de 1,2\%. La distribución del ingreso es muy desigual y la población debajo de la línea de pobreza era del 22\% en 2000 y la tasa de alfabetización fue de $89 \%$ en 2004.

Para 2004, según cifras derivadas de la PNAD, el índice de Gini de los ingresos monetarios era de 0.559. Tanto la desigualdad como la pobreza han tenido una lenta, pero continua disminución sobre todo a partir del 2000.

La evolución de las condiciones de empleo en Brasil estuvo caracterizada 
por un aumento notable del desempleo abierto a partir de la década del 90, aunque en los últimos años tendió a disminuir. Tiene una estructura industrial muy desarrollada y el PIB industrial representó el 30\% del PIB total en 2004. El ingreso laboral ha crecido lentamente, pero con una tendencia sostenida a partir de ese año.

En 2004, la población de México era de 102 millones de personas, en tanto el PIB fue de 6,834.9 millones de doláres. El ingreso per cápita fue de 6930 dólares. Al igual que el Brasil, entre 1990 y 2005, el PIB per cápita creció a una tasa media anual de 1,2\%. Para 2004, la población debajo de la línea de pobreza, representaba el $18 \%$ del total de habitantes del país, mientras que la tasa de alfabetización fue de $91 \%$ en ese mismo año.

Según cifras del Instituto Nacional de Estadística, Geografía e Informática (INEGI), en 2004 el coeficiente de Gini para los ingresos monetarios era de 0,541. La desigualdad y la pobreza tendieron a decrecer después del 2000, pero a partir de 2005 este proceso se estancó.

Las condiciones de empleo en México están caracterizadas por un bajo nivel de desempleo abierto, explicado por la presencia de un numeroso grupo de trabajadores por cuenta propia y una significativa migración internacional. Adicionalmente, las relaciones salariales estuvieron, en México, a niveles inferiores a las de Brasil antes de la década de los noventa, lo cual se traduce en que la insuficiencia de oportunidades ocupacionales se exprese más claramente en la forma de actividades de pequeña escala, y no en la de desempleo abierto como en el caso brasileño.

Su estructura industrial está muy desarticulada y el PIB sectorial es del 26\% del total. El ingreso laboral creció entre 1990 y 1994 para caer en 1995 y recuperarse lentamente, de manera que apenas en 2005 se alcanzaron los niveles medios observados en 1994 , antes de la crisis.

Los resultados obtenidos en el apartado anterior señalan una tendencia común a Brasil y a México de crecimiento en la tasa de actividad de las mujeres, de un abatimiento de la brecha de ingresos entre hombres y mujeres y de una mayor participación de las mujeres en actividades tipificadas como masculinas (como es el caso de la industria manufacturera). Estos resultados son, en cierto modo, esperables ya que los países examinados tienen rasgos estructurales en común. El que ambas naciones tengan un importante 
mercado externo (para 2004, el porcentaje de las exportaciones en el PIB era de $16 \%$ en Brasil y $30 \%$ en México), que su nivel de PIB sea semejante (el PIB de México fue solo 2.9\% mayor que el PIB de Brasil en 2004), que su estructura productiva sea de las más diversificadas de América Latina, y que una parte significativa de su producción esté volcada al mercado interno, son elementos que muestran la similitud a la que hacemos referencia. En estas condiciones de desarrollo capitalista también era de esperarse un proceso de búsqueda de independencia y empoderamiento por parte de las mujeres, sobre todo las jóvenes. Hay que señalar, sin embargo, que el aumento de la tasa de participación femenina en Brasil fue mucho más significativo en Brasil que en México.

Al mismo tiempo, el análisis de la estructura por sexos de la actividad económica, y de los cambios en la segregación por sectores apuntan a importantes diferencias, que son explicadas por la historia distinta de cada país, en términos de las especificidades de su desarrollo capitalista.

El más importante hallazgo de este estudio consiste en que la baja en la segregación de sexo por sectores de actividad fue más significativa en Brasil que en México (-0.793 contra -0.474). Esto se debe, especialmente al hecho de que en éste último país, la entrada de las mujeres a la actividad económica, ocurrió al mismo tiempo en las manufacturas y en actividades del sector comercio y servicios. El primero de estos sectores es más segregado que el sector de servicios, de modo que la baja total en la segregación fue reducida, ya que el número de mujeres que entraron en la manufactura fue casi la mitad del observado en los servicios y el comercio. En el caso del Brasil, si bien ocurrió un fenómeno semejante, el monto de mujeres que ingresó en las manufacturas fue tan significativo que cambió la estructura por sexo de esa actividad, mientras que este proceso no ocurrió en las actividades terciarias, donde la presencia de mujeres ya era significativa.

Por otro lado, la mayor participación de las mujeres en las actividades económicas en el Brasil, en comparación con México (52,9 y 35,2\%, respectivamente), puede ser una consecuencia de que las relaciones de asalariamiento en el Brasil, a pesar de que hoy en día estén en niveles totales comparables, estuvieron, históricamente, más expandidas en el caso de las mujeres de lo que ocurrió en México (KILM, 2007). 
Habria finalmente que destacar que las brechas entre el salario femenino y masculino en Brasil siguen más altas que las de México.

\section{Conclusiones}

La segregación sectorial e ocupacional por género consiste en un fenómeno que se encuentra en el corazón de las desigualdades de género en el mercado de trabajo. Frente a la creciente inserción de las mujeres en las actividades extradomésticas y de su mayor nivel de escolarización en relación a los hombres (una característica de prácticamente todas las sociedades industrializadas del occidente) las diferencias relacionadas a las condiciones de trabajo e a los salarios pasan a concentrarse cada vez más en la segregación sectorial y ocupacional.

Buscamos en este texto comparar las desigualdades de género en el mercado de trabajo brasileño y mexicano, teniendo como elemento central a la medición de la segregación sectorial por género. Para esto utilizamos el índice KM que nos reveló claramente las diferencias entre la segregación por género en los dos países. Además, la descomposición del índice entre dos momentos, permitió encontrar las causas subyacentes a los cambios en la segregación, las cuales son diferentes en cada país.

El análisis reveló que la inserción de las mujeres en la fuerza de trabajo en México es no solamente menos profunda como más segregada en actividades menos valorizadas, como las del sector terciario, cuando se compara con la inserción femenina en el caso brasileño.

Tales hallazgos nos plantean, sin embargo, una cuestión no trivial, para la cual el estudio aquí desarrollado no tiene respuestas: Si la segregación sectorial por género es más profunda en México que en Brasil, ¿cómo se explican las mayores brechas salariales entre hombres y mujeres en este último país?

A pesar de tratarse de un primer acercamiento al estudio comparativo de la segregación en ambos países, los resultados obtenidos son, en sí mismos, importantes. Pero a la vez apuntan a la necesidad de llevar a cabo un estudio más exhaustivo de las características del empleo femenino en cada país, a fin de identificar con más precisión aquello que nos es común y aquello que nos separa. 


\section{Referencias Bibliográficas}

ADLER, Leoneore (Ed.). International handbook on gender roles. Westport: Greenwood Press, 1993.

ANKER, Richard. Gender and jobs: sex segregation of occupations in the world. Ginebra: OIT, 1998.

BLACKBURN, Robert M.; SILTANEN, Janet; JARMAN, Jennifer. The measurement of occupational gender segregation: current problems and a new approach. Journal of the Royal Statistical Society, Series A, v. 158, n. p. 319-331, 1995.

BRUSCHINI, Cristina; LOMBARDI, Maria Rosa. Mulheres e homens no mercado de trabalho brasileiro: um retrato dos anos 1990. In: MARUANI, Margaret; HIRATA, Helena. As novas fronteiras da desigualdade. Homens e mulheres no mercado de trabalho. São Paulo: Editora Senac, 2003. p. 323361.

CACCIAMALI, Maria Cristina; HIRATA, Guilherme. Discriminação ou grupos em situação de desvantagem no mercado de trabalho. Uma análise do mercado de trabalho brasileiro acerca da raça e gênero. Revista de Economia Mundial, n. 12, p. 53-86, 2005.

CARLSON, Susan. Trends in race/sex occupational inequality: conceptual and measurement issues. Social Problems, v. 39, n. 3, p. 268-290, Ago. 1992.

DUNCAN, Otis; DUNCAN, Beverly. Analysis of segregation indexes. American Sociological Review, v. 20, n. 2, p. 210-217, 1955.

ENGLAND, Paula. Comparable worth. Theories and evidence. Nueva York: Aldine de Gruyter, 1992.

FOGEL, Miguel Nathan. The effects of gender segregation at the establishment level on wages: an empirical analysis using a panel of matched employer-employee data. Seminario en la EPGE, Fundação Getúlio Vargas, Oct. 2006

HAKIM, Catherine. A century of change in occupational segregation. 18911991. Journal of Historical Sociology, v. 7, n. 4, p. 435-454, Dec. 1994. 
JACOBS, Jerry. Evolving patterns of sex segregation. In: BERG, Ivar; KALLEBERG, Arne (Eds.). Sourcebook of labor markets: evolving structures and processes. New York: Kluwer Academic/Plenum Publishers, 2001.

KARMEL, Tom; MACLACHLAN, Maureen. Occupational sex segregation - increasing or decreasing? The Economic Record, p. 187-195, Sept. 1988.

MALDONADO, Victor; RENDÓN, Teresa. Evolución reciente del trabajo de hombres y mujeres en México. Comercio Exterior, v. 55, n. 1, p. 44-57, enero. 2005.

OIT. Global employment trends for women brief. Ginebra: International Labour Office, marzo. 2007.

OLIVEIRA, Ana María. H. Occupational Gender Segregation and Effects on Wages in Brazil. Proceedings of XXIV General Population Conference. International Union for the Scientific Study of Population, 2001.

POCHMANN, Marcio; SALAS, Carlos; LEITE, Marcia. Mercado de trabajo: ¿Qué hay de nuevo en Brasil? Revista Trabajo, México, UAM/Iztapalapa/ OIT, n. 4, enero/julio. 2007.

SALAS, Carlos. Segregación y discriminación laboral por sexo. México: Documento de Trabajo, Departamento de Sociología, UAM Iztapalapa, 2004.

VELLA, Francis. Gender roles, occupational choice and gender wage differential. Economic Record, v. 69, n. 207, p. 382-392, Dec. 1993.

WATTS, Martin. How should occupational sex segregation be measured? Work, Employment and Society, v. 6, n. 3, p. 475-487, 1992.

WATTS, Martin. Occupational gender segregation: index measurement and econometric modeling. Demography, v. 35, n. 4, p. 489-496, 1998. 